\title{
In Memory of Dr. James J. Mezhir, June 28, 1973-February 3, 2016
}

Published online: 27 April 2016

(C) 2016 The Society for Surgery of the Alimentary Tract

Dr. James J. Mezhir, Assistant Professor of Surgery in the Division of Surgical Oncology and Endocrine Surgery at the University of Iowa and a member of our Editorial Board, passed away on February 3, 2016, after a nearly 2-year battle with gastric cancer. Dr. James J. Mezhir was born on June 28, 1973, in Niagara Falls, New York. Dr. Mezhir's journey to becoming a physician is a unique experience in that he was a 25 -year survivor of cancer. When he was a teenager, James was diagnosed with non-Hodgkin's lymphoma after undergoing a Whipple procedure for bile duct obstruction. He underwent 12 months of chemotherapy and had been cancer free since that time. Being cured of cancer is what drove Dr. Mezhir toward caring for cancer patients and into the research field. He wanted to show children diagnosed with this disease that there is a future and life after cancer. Dr. Mezhir attended medical school at the State University of New York in Buffalo, New York, and he completed his residency at the University of Chicago under the training of Dr. Mitchell Posner and Dr. Jeffrey Matthews; at which time, he completed 2 years of dedicated basic science research in the laboratory of Dr. Ralph Weischselbaum. Dr. Mezhir continued his training as a fellow in Surgical Oncology at Memorial Sloan-Kettering Cancer Center in New York City where he worked closely with Dr. Ron DeMatteo and Dr. Murray Brennan. Dr. Mezhir was recruited to the University of Iowa in 2010, where he became a valued member of the
Division of Surgical Oncology and Endocrine Surgery in the Department of Surgery. As a surgical oncologist, his primary clinical practice was in the treatment of pancreatic cancer and other GI cancers. He collaborated with many basic scientists and clinicians in multiple disciplines in an effort to advance the field of pancreatic cancer research. Through his laboratory efforts, which focused on elucidating the complex biology of pancreatic and gastric cancer, Dr. Mezhir made novel discoveries in the biology of pancreatic and gastric cancer that will continue to have important translational implications that will eventually lead to novel therapies for these GI malignancies. Dr. Mezhir had over 50 peer-reviewed publications and was on the editorial board of six surgical and scientific journals, including ours. In addition to his role on the Editorial Board of the Journal of Gastrointestinal Surgery, James was an active member of the Society for Surgery of the Alimentary Tract. He and his wife, Priya George Mezhir, have two young daughters, Malinee and Anjali. He will be deeply missed by his peers, trainees, patients, and colleagues. We ask that you consider a donation to the "James Mezhir Memorial Fund" c/o Elizabeth Mezhir (mail to the Gay \& Ciha Funeral and Cremation Service (a) 2720 Muscatine Avenue, Iowa City, IA 52240, USA) which has been established for his daughters, Malinee and Anjali's education, or consider a donation in his memory to the Society for Surgery of the Alimentary Tract Foundation. 